\title{
LA PERCEPCIÓN DE LOS ALUMNOS COMO INDICADOR DE INCLUSIÓN EDUCATIVA
}

\author{
(STUDENTS' PERCEPTION AS AN INDICATOR OF EDUCATIONAL \\ INCLUSION)
}

Margarita Rodríguez Gudiño

Equipo de Orientación Educativa y Psicopedagógica (EOEP)

de Olivenza (Badajoz)

Cristina Jenaro Río

Universidad de Salamanca

Raimundo Castaño Calle

Universidad Pontificia de Salamanca

DOI: $10.5944 / e d u c X X 1.30198$

Cómo referenciar este artículo/How to reference this article:

Rodríguez Gudiño, M., Jenaro Río, C. y Castaño Calle, R. (2022). La percepción de los alumnos como indicador de inclusión educativa. Educación XX1, 25(1), 357-379. https://doi.org/10.5944/ educXX1.30198

Rodríguez Gudiño, M., Jenaro Río, C., \& Castaño Calle, R. (2022). Students' perception as an indicator of educational inclusion. Educación XX1, 25(1), 357-379. https://doi.org/10.5944/ educXX1.30198

\section{RESUMEN}

El principal objetivo de este estudio ha sido analizar la relación entre la percepción de los alumnos de sus centros educativos, en cuanto a centros inclusivos, y el estatus sociométrico de los mismos. Para ello se contó con una muestra de 881 alumnos pertenecientes a 43 aulas de primaria (de 6 a 12 años) de diferentes niveles educativos, entre los cuales se encontraban 98 alumnos con necesidades específicas de apoyo educativo $(11,12 \%)$, todos ellos escolarizados en centros educativos ordinarios de la región de Extremadura (España). Para analizar el estatus sociométrico se utilizó un cuestionario basado en el método de nominación de iguales y la asociación de atributos. Para valorar la percepción de los alumnos de sus centros educativos se utilizó el Cuestionario sobre el Punto de Vista de los Niños extraído del 
Index for Inclusion (Booth, Ainscow y Kingston, 2006). El estudio mostró que la percepción que tienen los alumnos de sus centros educativos, tanto si estos tienen necesidades específicas de apoyo educativo como si no, está significativamente asociada a su estatus sociométrico, y más concretamente, al rechazo que reciben en sus clases por parte de sus iguales. También indicó que los estudiantes con necesidades específicas de apoyo educativo se ven más apoyados por los profesores que por sus compañeros. Todo ello nos lleva a reflexionar sobre si las medidas organizativas que adoptan las escuelas para dar respuesta a las necesidades educativas puedan afectar a su inclusión. De ahí, la necesidad de evaluar periódicamente las estrategias de agrupamiento y la percepción de los alumnos de sus centros educativos.

\section{PALABRAS CLAVE}

Percepción de los alumnos, aceptación de iguales, técnicas sociométricas, inclusión, estudiantes con necesidades educativas especiales, primaria

\section{ABSTRACT}

The main goal of this study was to analyze the relationship between pupils' perception of their schools as inclusive schools and their sociometric status. For this, a sample of 881 students belonging to 43 primary classrooms (6 to 12 years old) of different educational levels, of which 98 pupils presented specific educational support needs $(11.12 \%)$, all of them enrolled in ordinary schools in the region of Extremadura (Spain). A questionnaire, based on the peers nomination method and attributes association, was used to analyze sociometric status. The Questionnaire on the Children's Point of View, taken from the Index for Inclusion (Booth, Ainscow \& Kingston, 2006), was used to assess the pupils' perception of their schools. The study showed that the perception that pupils have of their schools, whether they have specific needs for educational support or not, is significantly associated with their sociometric status, and more specifically, the rejection they receive in their classrooms from their peers. The results also indicated that students with specific educational needs feel more supported by teachers than by their peers. This leads us to reflect on whether the organizational measures adopted by schools affect their inclusion. Hence, the need to periodically assess grouping strategies and pupils' perception of their schools.

\section{KEY WORDS}

Student evaluation, peer acceptance, sociometric techniques, inclusion, special needs students, elementary schools 


\section{INTRODUCCIÓN}

La inclusión educativa se ha considerado como la mejor práctica en educación durante muchos años (Stites, Rakes, Noggle y Sha, 2018). Esta va más allá del mero emplazamiento de estudiantes con necesidades educativas especiales en clases ordinarias, requiere también eliminar barreras para la participación (Schwab, 2018). Se puede entender como un asunto metodológico y organizativo que afecta a diferentes niveles dentro del sistema educativo para lograr objetivos educativos sociales como la justicia social y participación de todos sus actores (Haug, 2017). Elementos clave para la educación inclusiva son la presencia, ubicación, participación y logros de todos los estudiantes (Ainscow, 2016). Cuando hablamos de todos los estudiantes, nos estamos refiriendo tanto a los que no tienen necesidades específicas como a quienes presentan dichas necesidades. Cabe recordar que según la Ley Orgánica de Educación (LOE, 2/2006, de 3 de mayo), el término de Necesidades Específicas de Apoyo Educativo (NEAE) engloba a alumnos con necesidades educativas especiales (NEE), con altas capacidades, con dificultades específicas de aprendizaje (p.ej. dislexia, discalculia) o que requieren medidas de tipo compensatorio generalmente por circunstancias sociales (p.ej. migrantes con incorporación tardía al sistema educativo). A su vez, dentro del término de NEE se incluye a alumnos cuyas necesidades se relacionan con condiciones personales de tipo físicas, psíquicas, cognitivas, sensoriales o de conducta. Los alumnos con discapacidad intelectual o con trastorno del espectro autista (TEA) se incluyen en este último término.

Muchos estudios muestran que una escuela inclusiva se caracteriza por su habilidad para trabajar como equipo cohesionado (Ainscow, 2016). Sin embargo, el éxito de la educación inclusiva no radica en enviar a los alumnos con necesidades especiales a escuelas ordinarias y esperar a que sucedan milagros, sino que depende ampliamente de la colaboración de padres y profesores (Adams, Harris y Jones, 2016). El profesor debe además garantizar la facilitación de experiencias de aprendizaje diarias de todos sus estudiantes. En consecuencia, los ambientes educativos deben ser diseñados atendiendo a las necesidades de los estudiantes, de modo que se maximice su participación con iguales en actividades académicas y no académicas. Esto contrasta con los datos que muestran que los estudiantes con NEE que asisten a centros ordinarios son emplazados en aulas especiales donde pueden llegar a pasar hasta más de un $20 \%$ de tiempo (Ramberg y Watkins, 2020). La brecha entre la teoría y la práctica de la inclusión es también destacada en recientes estudios (Moliner, Arnaiz y Sanahuja, 2020).

Las relaciones e interacciones sociales juegan un rol importante en el desarrollo saludable del niño y de su socialización (Perolli-Shehu, 2019) y el grupo de iguales tiene una importancia excepcional al respecto (Krampač 
y Kolak, 2018), pues mediante el grupo-clase se crea un grupo formal de iguales (Krampač y Kolak, 2018). Por ello, es importante analizar cómo los alumnos en general y con necesidades educativas especiales, en particular, perciben su aceptación por iguales con o sin necesidades educativas, y cuál es su percepción de pertenencia al ambiente ordinario de aprendizaje. Aceptación y pertenencia son conceptos estrechamente asociados que impactan e influyen el uno sobre el otro, como varios estudios sugieren (Rose, Barahona y Muro, 2017; Rose y Shevlin, 2017).

De acuerdo con los planteamientos de Crouch y col., (Crouch, Keys y McMahon, 2014), la percepción de aceptación por parte de los alumnos con necesidades educativas especiales o discapacidad, se relaciona con sus experiencias de interacciones positivas con otros. Así pues, donde se sienten respetados y tratados de forma similar a sus iguales, perciben aceptación por parte del grupo y pertenencia a dicho ambiente. En esta línea, se ha mostrado que los alumnos que no reciben apoyo fuera de su aula habitual son más aceptados que aquellos estudiantes que salen de la misma para recibir apoyos especiales (Rose et al., 2017). Varios estudios muestran que la participación e interacción social de niños con y sin NEE está condicionada por las características del niño y la forma de organización educativa (Rose et al, 2017; Wendelborg y Tøssebro, 2011). En estos estudios se sugiere que no es en sí la NEE lo que genera diferencias, sino el tipo de apoyo educativo que se recibe y el grado de participación en clase (Wendelborg y Tøssebro, 2011). Cabe también señalar que las NEE más significativas se asocian a más horas fuera del aula (Wendelborg y Tøssebro, 2011). Esto es relevante porque el estatus social del niño implica el fenómeno de aceptación o rechazo por su grupo de iguales. Así, los alumnos que no se sienten apreciados o se sienten rechazados por otros se frustran, aburren y dejan de participar en las actividades de aprendizaje (Prince y Hadwin, 2013).

La tradición perceptual y la teoría social cognitiva reconocen la importancia de las percepciones para comprender y predecir la conducta individual (Purkey y Novak, 2016). Las percepciones se relacionan con el modo en que pensamos acerca de los demás y de nosotros mismos. Dichas percepciones tienen además la capacidad de guiar nuestra conducta, pues tendemos a reaccionar a las experiencias en función de cómo las percibimos. Estudios previos mostraron que la percepción de los estudiantes son un reflejo y producto del clima de la escuela, y se diferencian según las características y experiencias de cada estudiante (Ng y Yuen, 2011). Sin embargo, y como señalan algunos autores (Schwab, Sharma y Hoffmann, 2019), las percepciones de estudiantes con y sin discapacidad han sido escasamente estudiadas. Contamos, sin embargo, con algunas excepciones. Por ejemplo, se ha explorado la relación entre la percepción del ambiente escolar y los resultados académicos o entre la percepción de la cultura 
escolar, la inclusión y las prácticas educativas por parte de estudiantes con y sin discapacidad (Shogren, Gross, Forber-Pratt, Francis, Satter, Blue-Banning y Hill, 2015). También se ha evaluado la percepción, por parte de los estudiantes, de prácticas inclusivas como la diferenciación y personalización (Lindner, Alnahdi, Wahl y Schwab, 2019), o la percepción de los iguales sin discapacidad hacia sus compañeros con discapacidad en contextos escolares y universitarios (Akin y Huang, 2019). Estos estudios son, a todas luces, escasos.

En esta línea, el presente estudio realizado con una muestra amplia de estudiantes de educación primaria explora la percepción de alumnos de sus centros educativos y su relación con la aceptación y rechazo que reciben por parte de sus iguales. Como se ha realizado en estudios previos (Rose et al., 2017), analizamos el estatus sociométrico de estudiantes de escuelas ordinarias que reciben apoyo educativo fuera del aula por diversas razones, pero además, añadimos como elemento innovador la exploración de las percepciones sobre sus centros educativos. Este aspecto nos ofrece datos especialmente valiosos de los estudiantes como informantes privilegiados de las interacciones y estructura del aula (Wallace, Kelcey y Ruzek, 2016). La identificación de las características y elementos asociados a las diferentes experiencias y percepciones de los estudiantes en relación a sus emplazamientos escolares debe ser objeto de estudio a nivel nacional e internacional (Ramberg y Watkins, 2020).

Por lo tanto, los objetivos que planteamos son: (1) Analizar el estatus sociométrico de los alumnos en función de su pertenencia o no al grupo de alumnos con necesidades específicas de apoyo educativo (ACNEAE). (2) Analizar la percepción que los alumnos con distinto tipo sociométrico tienen de sus centros educativos. (3) Analizar diferencias y semejanzas dependiendo de si los alumnos forman parte del grupo de alumnos con necesidades específicas de apoyo educativo o no. Además, esperamos encontrar:

1. Mayores niveles de aceptación en alumnos sin NEAE y mayores niveles de rechazo en alumnos con NEAE.

2. Mayores niveles de satisfacción con el centro educativo por parte de los niños que son aceptados (populares o entrañables).

3. Niveles significativamente inferiores de satisfacción con el centro educativo por parte de niños que no experimentan tal aceptación o que son rechazados (olvidados o rechazados). 
4. Estas diferencias se mantendrán tanto para alumnos con necesidades específicas de apoyo educativo, como por quienes no las presentan.

\section{MATERIALES Y MÉTODO}

\section{Participantes}

La muestra estuvo conformada por 881 alumnos de primaria, de edades comprendidas entre los 6 y 12 años, y de diferentes niveles educativos, escolarizados en centros de diversas localidades. La muestra representa a 20 centros educativos ordinarios, de los cuales el $82.4 \%$ eran de titularidad pública, y el 17.6\% de titularidad concertada. Según su ubicación geográfica, el 70\% se encontraban en la capital de la provincia, el 25\% en zonas urbanas, y el 5\% en zonas rurales.

Se adoptó como criterio de inclusión de los alumnos en el estudio la aceptación de los centros a participar, la autorización y consentimiento informado de sus padres, y la participación voluntaria de sus profesorestutores y los alumnos. En la Tabla 1 se muestra la distribución de los 881 alumnos por niveles. Se puede apreciar cómo un total de 98 alumnos (11.12\%) presentaban necesidades específicas de apoyo educativo (NEAE). Todos estos estudiantes asisten a aulas ordinarias o grupos-clase de referencia, si bien, reciben apoyo fuera del aula como medida de atención a la diversidad.

Entre los alumnos con NEAE predominaban los alumnos con trastorno por hiperactividad con déficit de atención (51\%), seguidos de discapacidad intelectual (16.3\%), dificultades del aprendizaje, inteligencia límite y desfase curricular significativo (15.3\%), trastorno del espectro autista $(6.1 \%)$, trastorno del lenguaje (4\%), trastorno grave de conducta $(2 \%)$, alta capacidad $(2 \%)$, discapacidad física $(1 \%)$, discapacidad auditiva (1\%) y discapacidad visual (1\%). Como se puede apreciar, este estudio incluye a distintos grupos de NEAE porque el grupo de alumnos con NEAE que cursan primaria es igualmente heterogéneo.

\section{Tabla 1}

Distribución de alumnos por niveles y por porcentajes de necesidades específicas de apoyo educativo (NEAE)

\begin{tabular}{cccc}
\hline Primaria & N & NEAE & $\%$ \\
\hline Primero & 36 & 4 & 11.11 \\
\hline Segundo & 141 & 16 & 11.35 \\
\hline Tercero & 205 & 20 & 9.76 \\
\hline
\end{tabular}




\begin{tabular}{cccc}
\hline Primaria & $\mathbf{N}$ & NEAE & $\%$ \\
\hline Cuarto & 218 & 22 & 10.09 \\
\hline Quinto & 156 & 19 & 12.18 \\
\hline Sexto & 125 & 17 & 13.60 \\
\hline Total & 881 & 98 & 11.12 \\
\hline
\end{tabular}

\section{Procedimiento}

Los datos fueron recogidos de enero a junio de 2019 de los 20 centros educativos participantes. Los sociogramas fueron aplicados a los alumnos en sus clases por sus profesores tutores, así como el Cuestionario sobre el Punto de Vista de los Niños (original de Booth, Ainscow y Kingston, 2006, traducción y adaptación al castellano por González-Gil, Gómez-Vela y Jenaro, 2007). Se proporcionaron instrucciones y formación para garantizar la adecuada recogida de datos y la confidencialidad de la información. El estudio fue aprobado con la Consejería de Educación del gobierno regional.

\section{Instrumentos}

Se elaboró un sociograma en el que se combinó el método de nominación de iguales con el método de asociación de atributos basado en estudios previos (Martín, Muñoz de Bustillo y Pérez, 2011). El cuestionario sociométrico se diseñó atendiendo a un solo criterio (preferencia), dos dimensiones (positiva y negativa) y dos contextos (clase y patio) (Nepi, Fioravanti, Nannini y Peru, 2015). Se optó por el procedimiento con tres elecciones máximas por contar con evidencias que este criterio aumenta la precisión frente a los basados en cinco elecciones (Baydik y Bakkaloglu, 2009; García-Bacete, 2006).

Con el método de asociación de atributos se obtiene información sobre cómo perciben la conducta los compañeros de clase. Siguiendo el modelo de Díaz-Aguado (2006) con la pregunta-estímulo: “¿Quién es el chico o chica de tu clase que destaca por...?", se utilizaron los siguientes atributos como estímulos: "Tener muchos amigos"; "Tener pocos amigos"; "Participar en todas las actividades"; "Participar en pocas actividades"; "Saber mucho"; "Saber poco"; "Necesitar mucha ayuda de los profesores"; "Necesitar poca ayuda de los profesores"; "Portarse como una persona madura"; "Portarse como un niño más pequeño"; "El que más molesta a los demás"; "El que menos molesta a los demás". Los análisis nos permitieron clasificar a los alumnos en función de su estatus sociométrico en cuatro tipos sociométricos: Rechazado, Olvidado, Entrañable o Popular. La asignación de los alumnos 
a cada tipo sociométrico se realizó tomando como referencia la propuesta de DeRosier y Thomas (2003). Así, los cuatro grupos fueron definidos como sigue: (1) Populares son aquellos alumnos que presentan puntuaciones altas en su estatus de Elección y puntuaciones bajas o medias en su estatus de Rechazo. (2) Entrañables son los alumnos que obtienen puntuaciones medias en Elección y bajas o medias en Rechazo. (3) Olvidados son los alumnos que obtienen valoración baja en Elección y valoración baja en Rechazo. (4) Rechazados, son aquellos alumnos que obtienen valoración baja o media en Elección y valoración alta en Rechazo.

Además, utilizamos el Cuestionario sobre el Punto de Vista de los Niños extraído del Index for Inclusion, Developing Play, Learning and Participation in Early Years and Childcare (Booth, Ainscow y Kingston, 2006). Concretamente, se trata del Cuestionario tres, que consta de 13 ítems a los que los alumnos responden con tres categorías de respuestas: "Siempre", "A veces", "Nunca". Estas categorías fueron añadidas dado que en el cuestionario original no se ofrece formato alguno de respuesta. De este modo hemos empleado el sistema de respuesta de tres puntos que se emplea en los restantes cuestionarios incluidos en el Index y destinado a niños de más edad o a otros informantes (padres, profesores, etc.). Mediante la respuesta a estos ítems se intenta conocer lo que los niños piensan sobre sus colegios. Se les pide que respondan con sinceridad expresando sus opiniones y no pensando en lo que pueda agradar a sus padres o profesores (González-Gil, Gómez-Vela y Jenaro, 2007). Puntuaciones más elevadas denotan una mayor satisfacción con el centro educativo. La fiabilidad obtenida en este instrumento fue de alfa $=.68$. En el grupo con NEAE la fiabilidad fue de alfa $=.76$, mientras que en el grupo sin NEAE la fiabilidad fue de alfa $=.67$. Puede considerarse que para ambos grupos y teniendo en cuenta la diversidad de contenido que engloba el cuestionario, los niveles de fiabilidad fueron adecuados.

\section{DISEÑO Y ANÁLISIS}

El presente estudio es de tipo exploratorio, descriptivo-transversal, y con medidas ex post facto. Para dar respuesta a los objetivos se han empleado estadísticos no paramétricos como la prueba Chi cuadrado y el análisis de los residuos tipificados corregidos (RC). Dado que estos residuos se distribuyen normalmente, cuando estos tienen un valor superior a 1.96 indican casillas con más casos de los que debiera haber en esas casillas si las variables estudiadas fueran independientes, mientras que los residuos menores de -1.96 indican casillas con menos casos de los que cabría esperar bajo la condición de independencia. Para el contraste de hipótesis y analizar la significatividad de las diferencias se ha empleado Anova. 


\section{RESULTADOS}

\section{Presencia o ausencia de NEAE y estatus sociométrico}

En relación al primer objetivo hemos analizado el porcentaje de alumnos que se encuentran en cada uno de los tipos sociométricos y si presentan NEAE o no las presentan. El análisis de la asociación reveló asociación significativa entre ambas variables (Chi cuadrado $=83.779$, $\mathrm{gl}=3, \mathrm{p}<0.001)$. El análisis de los residuos corregidos mostró que para los estudiantes sin NEAE es mucho más probable recibir valoración sociométrica de Entrañable ( $\mathrm{RC}=6.1$ ) o Popular (3.5) y menos probable de Rechazado $(\mathrm{RC}=-5.9)$ u Olvidado $(\mathrm{RC}=-5.7)$. En cambio, el análisis mostró que para los estudiantes con NEAE es mucho menos probable recibir valoración sociométrica de Entrañable ( $\mathrm{RC}=-6.1)$ o Popular $(\mathrm{RC}=-3.5)$, mientras que es mucho más probable ser Rechazado $(\mathrm{RC}=5.9)$, u Olvidado $(\mathrm{RC}=5.7)$. En la Figura 1 se presentan los porcentajes obtenidos en cada caso. Se puede apreciar cómo casi el 50\% de los alumnos con necesidades específicas de apoyo educativo pertenecen al grupo olvidado. Por su parte, más del 55\% de los alumnos sin dichas necesidades pertenecen al grupo entrañable.

\section{Figura 1}

Porcentaje de alumnos categorizados como entrañables, olvidados, populares o rechazados desagrupados por presencia o ausencia de necesidades específicas de apoyo educativo

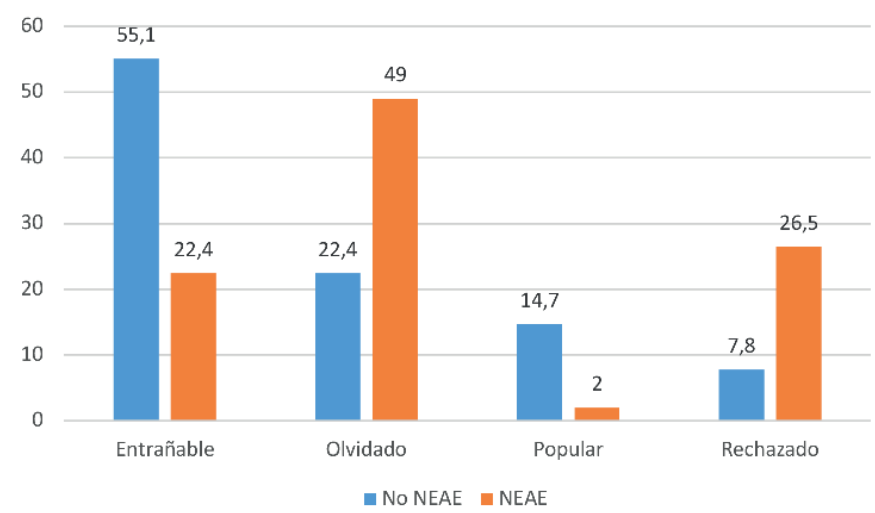

\section{Tipo sociométrico y percepción del centro educativo}

En relación al objetivo 2 de este estudio analizamos la posible existencia de diferencias en los ítems del cuestionario así como en el cuestionario considerado globalmente sobre la muestra total de alumnos. En la Tabla 2 se puede apreciar la existencia de diferencias significativas en los ítems 
2, 3, 6, 8 y total. Los análisis post hoc (Duncan) revelaron que en los ítems 2,3 y 8 , los alumnos rechazados obtienen puntuaciones significativamente más bajas que los demás compañeros. En el ítem 5 los alumnos rechazados puntúan significativamente más bajo que los alumnos entrañables. En el ítem 6 los alumnos populares puntúan significativamente más elevado que los restantes grupos.

Los resultados en cuanto a satisfacción de los alumnos con sus colegios no parecen estar mediados ni por sus logros (ítem 11), ni con la percepción que las familias tienen de los centros educativos (ítem 13).

\section{Tabla 2}

Estadísticos descriptivos y significación de las diferencias (Anova) en función de los tipos sociométricos

\begin{tabular}{ccccccc}
\hline & N & Media & SD & SE & F & Sig. \\
\hline it1_Me gusta venir al colegio & & & & & 1.704 & 0.165 \\
\hline Entrañable & 443 & 2.47 & 0.58 & 0.03 & & \\
\hline Olvidado & 210 & 2.57 & 0.60 & 0.04 & & \\
\hline Popular & 115 & 2.50 & 0.58 & 0.05 & & \\
\hline Rechazado & 87 & 2.54 & 0.55 & 0.06 & & \\
\hline it2_Me gusta jugar con mis amigos & & & & & 8.02 & 0.000 \\
\hline Entrañable & 444 & 2.88 & 0.34 & 0.02 & & \\
\hline Olvidado & 210 & 2.81 & 0.43 & 0.03 & & \\
\hline Popular & 116 & 2.93 & 0.25 & 0.02 & & \\
\hline Rechazado & 87 & 2.70 & 0.55 & 0.06 & & \\
\hline it3_A mis amigos les gusta jugar conmigo & & & & 7.94 & 0.000 \\
\hline Entrañable & 437 & 2.70 & 0.49 & 0.02 & & \\
\hline Olvidado & 205 & 2.56 & 0.56 & 0.04 & & \\
\hline Popular & 114 & 2.66 & 0.55 & 0.05 & & \\
\hline Rechazado & 86 & 2.43 & 0.66 & 0.07 & & \\
\hline Entrañable & 439 & 2.58 & 0.56 & 0.03 & & \\
\hline Olvidado & 205 & 2.53 & 0.59 & 0.04 & & \\
\hline Popular & 113 & 2.55 & 0.60 & 0.06 & & \\
\hline Rechazado & 86 & 2.45 & 0.63 & 0.07 & & \\
\hline Entrañable & 428 & 2.76 & 0.47 & 0.02 & & \\
\hline it5_A mis profesores les gusta ayudarme & & & & \\
\hline & & & & & \\
\hline
\end{tabular}




\begin{tabular}{|c|c|c|c|c|c|c|}
\hline & $\mathbf{N}$ & Media & SD & SE & $\mathbf{F}$ & Sig. \\
\hline Olvidado & 199 & 2.70 & 0.56 & 0.04 & & \\
\hline Popular & 112 & 2.70 & 0.53 & 0.05 & & \\
\hline Rechazado & 78 & 2.62 & 0.59 & 0.07 & & \\
\hline \multicolumn{5}{|c|}{ it6__ Me gusta ayudar a los profesores } & 2.783 & 0.040 \\
\hline Entrañable & 443 & 2.53 & 0.65 & 0.03 & & \\
\hline Olvidado & 203 & 2.50 & 0.66 & 0.05 & & \\
\hline Popular & 116 & 2.68 & 0.49 & 0.05 & & \\
\hline Rechazado & 85 & 2.45 & 0.70 & 0.08 & & \\
\hline \multicolumn{5}{|c|}{ it7_Algunos niños insultan a otros } & 0.381 & 0.767 \\
\hline Entrañable & 441 & 2.15 & 0.49 & 0.02 & & \\
\hline Olvidado & 209 & 2.16 & 0.63 & 0.04 & & \\
\hline Popular & 114 & 2.10 & 0.55 & 0.05 & & \\
\hline Rechazado & 87 & 2.16 & 0.59 & 0.06 & & \\
\hline \multicolumn{5}{|c|}{ it8_ A veces los niños no son muy amables conmigo } & 4.565 & 0.004 \\
\hline Entrañable & 429 & 2.56 & 0.52 & 0.03 & & \\
\hline Olvidado & 202 & 2.47 & 0.53 & 0.04 & & \\
\hline Popular & 111 & 2.50 & 0.55 & 0.05 & & \\
\hline Rechazado & 85 & 2.33 & 0.64 & 0.07 & & \\
\hline \multicolumn{5}{|c|}{ it9_Cuándo me siento triste siento que algún adulto me ayuda } & 0.447 & 0.720 \\
\hline Entrañable & 442 & 2.62 & 1.58 & 0.08 & & \\
\hline Olvidado & 207 & 2.50 & 0.71 & 0.05 & & \\
\hline Popular & 114 & 2.57 & 0.61 & 0.06 & & \\
\hline Rechazado & 86 & 2.52 & 0.68 & 0.07 & & \\
\hline \multicolumn{5}{|c|}{$\begin{array}{l}\text { it10_Cuando los niños se pelean el profesor sabe cómo } \\
\text { arreglarlo }\end{array}$} & 0.474 & 0.701 \\
\hline Entrañable & 444 & 2.76 & 0.50 & 0.02 & & \\
\hline Olvidado & 207 & 2.73 & 0.52 & 0.04 & & \\
\hline Popular & 112 & 2.70 & 0.53 & 0.05 & & \\
\hline Rechazado & 84 & 2.76 & 0.51 & 0.06 & & \\
\hline \multicolumn{5}{|c|}{ it11_Me siento satisfecho con mis logros } & 1.385 & 0.246 \\
\hline Entrañable & 440 & 2.77 & 0.47 & 0.02 & & \\
\hline Olvidado & 207 & 2.73 & 0.53 & 0.04 & & \\
\hline
\end{tabular}




\begin{tabular}{|c|c|c|c|c|c|c|c|}
\hline & & $\mathbf{N}$ & Media & SD & SE & $\mathbf{F}$ & Sig. \\
\hline & Popular & 112 & 2.69 & 0.60 & 0.06 & & \\
\hline & Rechazado & 86 & 2.66 & 0.64 & 0.07 & & \\
\hline \multicolumn{6}{|c|}{$\begin{array}{l}\text { it12_A mis profesores les gusta que les cuente lo que hago } \\
\text { en casa }\end{array}$} & 0.222 & 0.881 \\
\hline & Entrañable & 437 & 2.20 & 0.71 & 0.03 & & \\
\hline & Olvidado & 206 & 2.23 & 0.75 & 0.05 & & \\
\hline & Popular & 111 & 2.22 & 0.72 & 0.07 & & \\
\hline & Rechazado & 85 & 2.15 & 0.79 & 0.09 & & \\
\hline \multicolumn{6}{|c|}{ it13_Mi familia piensa que este es un buen colegio } & 1.661 & 0.174 \\
\hline & Entrañable & 432 & 2.88 & 0.36 & 0.02 & & \\
\hline & Olvidado & 202 & 2.87 & 0.38 & 0.03 & & \\
\hline & Popular & 111 & 2.95 & 0.23 & 0.02 & & \\
\hline & Rechazado & 83 & 2.84 & 0.40 & 0.04 & & \\
\hline \multirow[t]{5}{*}{ TOTAL } & & & & & & 2.65 & 0.048 \\
\hline & Entrañable & 447 & 31.81 & 3.57 & 0.17 & & \\
\hline & Olvidado & 212 & 31.14 & 4.26 & 0.29 & & \\
\hline & Popular & 116 & 31.77 & 3.41 & 0.32 & & \\
\hline & Rechazado & 87 & 30.89 & 3.40 & 0.36 & & \\
\hline
\end{tabular}

\section{Percepción del centro y estatus sociométrico de alumnos sin NEAE}

En relación al objetivo 3 se han realizado los mismos análisis desglosando si presentan o no NEAE. Así, y respecto a los alumnos que no presentan NEAE, la Tabla 3 resume los resultados obtenidos en los ítems donde se encontraron diferencias significativas. Los análisis post hoc (Duncan) revelaron que en los ítems 2, 3 y 8, los alumnos rechazados obtienen puntuaciones significativamente más bajas que los demás compañeros. En el ítem 5 los alumnos rechazados puntúan significativamente más bajo que los alumnos entrañables. En el ítem 6 los alumnos rechazados puntúan significativamente más bajo que los entrañables y los populares. 


\section{Tabla 3}

Estadísticos descriptivos y significación de las diferencias (Anova) en función de los tipos sociométricos. en alumnos sin NEAE

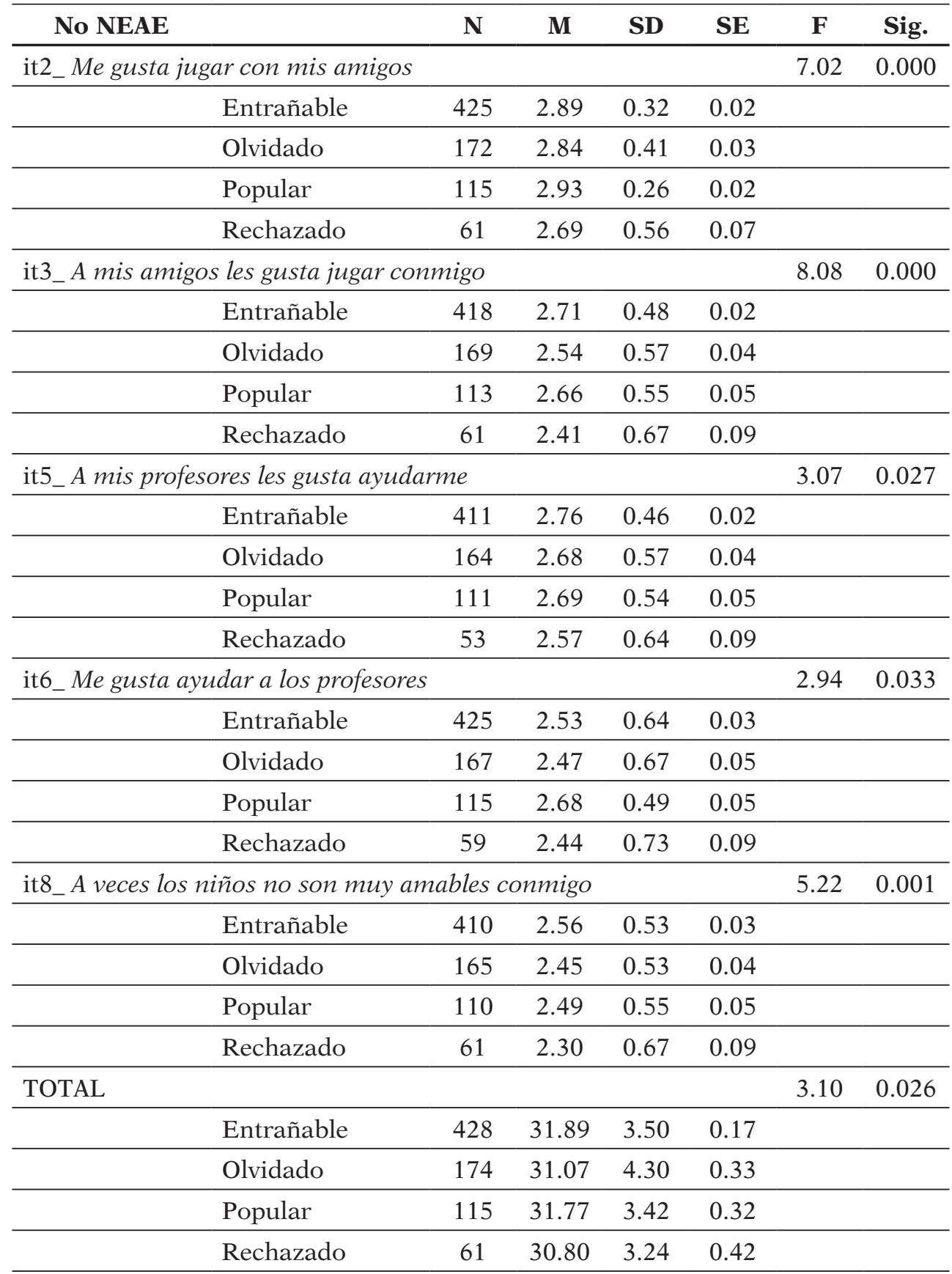




\section{Percepción del centro por parte de los alumnos con NEAE}

Los análisis realizados con el grupo de Alumnos con Necesidades Específicas de Apoyo Educativo no revelaron diferencias estadísticamente significativas entre los tipos sociométricos. Es importante señalar que una limitación hallada en relación a los análisis estadísticos realizados es el tamaño muestral de algunos de los tipos sociométricos. Por ello, el tipo sociométrico Popular $(\mathrm{N}=1)$ tuvo que ser eliminado de los análisis por ser no representativo.

\section{Diferencias en la percepción del centro entre alumnos con y sin NEAE}

Por último, los análisis realizados comparando separadamente a cada grupo sociométrico en función de la presencia o ausencia de NEAE puso de manifiesto que en el grupo Entrañable no se encontraron diferencias significativas en la valoración del centro, ni globalmente ni en ninguno de los ítems. Los análisis con el grupo Olvidado revelaron diferencias significativas entre alumnos con o sin NEAE en los ítems 4 'A mis profesores les gusta escucharme', y 12 'A mis profesores les gusta que les cuente lo que hago en casa' y los alumnos con NEAE puntuaron significativamente más elevado. Los análisis con el grupo rechazado tampoco revelaron diferencias significativas. Por último, y como señaláramos anteriormente, no fue posible realizar análisis con el grupo Popular por la ausencia de alumnos con NEAE que se incluyeran en esta categoría.

\section{DISCUSIÓN}

El presente estudio ofrece evidencias que confirman la menor aceptación por parte de iguales de compañeros con necesidades educativas. Estos resultados están en consonancia con los obtenidos de estudios previos que muestran cómo los alumnos con discapacidad son menos aceptados que sus compañeros (Baydik y Bakkaloglu 2009; Héctor y Olivarez,1995; Nepi et al., 2015). Así, de acuerdo con nuestra primera hipótesis se confirma que los alumnos con NEAE son predominantemente categorizados como Rechazados u Olvidados, mientras que los alumnos que no presentan NEAE son predominantemente categorizados como Populares o Entrañables. Cabe señalar que en el presente estudio, cuando hacemos referencia a Rechazado estamos refiriéndonos a ser "sociométricamente rechazado". Esto es, se consideran rechazados aquellos alumnos que obtienen un estatus de rechazo superior a la media de la clase y un estatus de elecciones por debajo de dicha media (García-Bacete, 2006; Pijl, Frostad y Flem, 2008). Además, 
mediante la técnica de nominación de iguales basada en el doble análisis (Like to Work and Like to Play), se considera que tan solo los niños elegidos como compañeros preferidos tienen una buena posición social (Nepi et al., 2015). En cualquier caso, los presentes hallazgos justifican la indagación sobre factores que pudieran estar en la base de esta desventaja en cuanto a preferencia social.

$\mathrm{Al}$ respecto, actualmente el interés investigador se centra no solo en analizar la aceptación o rechazo hacia los alumnos con NEE, sino también los factores que conducen a tal aceptación o rechazo por parte de los iguales (Perolli-Shehu, 2019). Estudios anteriores han analizado factores personales como las habilidades lingüísticas (Van der Wilt, Van der Veen, Van Kruistum y Van Oers, 2020), la competencia social y emocional (Blandon, Calkins, Grimm, Keane y O'Brien, 2010) o la presencia de discapacidad (Baydik y Bakkaloglu 2009; Kwon, Hong y Jeon, 2017). También han puesto de manifiesto el peso de las actitudes de los iguales y el impacto del contexto según la presencia o no de una unidad de educación especial en las escuelas (Vignes, Godeau, Sentenac, Coley, Navarro, Grandjean y Arnaud, 2009), las estrategias de agrupamiento (De Vroey, Struyf y Petry, 2016 ) o las estrategias organizativas que adoptan las escuelas para responder a los alumnos con NEAE mediante atención especializada fuera del aula (Rose et al., 2017; Wendelborg y Tøssebro, 2011).

Los presentes hallazgos, obtenidos con un grupo amplio y diverso de alumnos con necesidades educativas, ofrecen apoyo a la preocupación de quienes advierten que la respuesta a la atención a la diversidad consistente en ofrecer apoyo fuera del aula para responder a las necesidades específicas de los estudiantes puede disminuir su participación en actividades donde sus iguales están implicados (Rose et al., 2017; Wendelborg y Tøssebro, 2011). Esta reducción de la participación y por tanto en el contacto, podría explicar la menor preferencia hacia estos alumnos. Las relaciones de compañerismo, afecto y amistad se construyen sobre la presencia y participación, y una reducción de estas puede a su vez tener consecuencias negativas en las relaciones.

El presente estudio pone de manifiesto que los alumnos que se sienten más aceptados por sus iguales, concretamente, los categorizados sociométricamente como Entrañables y Populares, experimentan una mayor satisfacción con el centro educativo. Lo contrario sucede con los categorizados como Olvidados y Rechazados que muestran menos satisfacción con dicho centro. Estos datos son especialmente relevantes, puesto que se trata de alumnos de primaria y los datos han sido obtenidos con dos instrumentos y técnicas diferentes, lo que aumenta la confianza en los presentes resultados. 
Los resultados muestran que los alumnos que reciben más rechazo disfrutan menos jugando con sus amigos (ítem 2), y perciben que a sus amigos les gusta menos jugar con ellos (ítem 3). Además, perciben que a veces sus compañeros no son amables con ellos (ítem 8). Por otra parte, no se han encontrado diferencias en cuanto a la percepción que tienen sobre la atención que reciben de sus profesores (ítems 4, 9, 10, 12) y los tipos sociométricos. Sin embargo, los alumnos Rechazados muestran menos interés por ayudar a sus profesores, mientras que los Populares manifiestan un interés significativamente más elevado. A ello se une el hecho de que cuando se analiza separadamente la situación de alumnos sin NEAE clasificados como Rechazados, también coinciden en percibir que a sus profesores les gusta menos ayudarles (ítem 5). Estos resultados presentan similitudes con un estudio realizado en el Reino Unido en el que, a partir de preguntas planteadas en el Index para la inclusión, se pidió a alumnos con y sin NEE que valoraran su acuerdo hacia enunciados del tipo: "me siento contento en este colegio", o "los profesores ayudan a todos los alumnos y no solo a los más capaces". Los análisis revelaron que los alumnos con NEE ofrecieron inferiores valoraciones (Hodson et al., 2005). En esta línea, en un estudio realizado en los Emiratos Árabes en el que se preguntaba a estudiantes con y sin NEE acerca de la inclusión de compañeros con discapacidad, se aprecia cómo en cursos iniciales la aceptación de estos alumnos es más elevada y también lo es la satisfacción de dichos alumnos con el colegio, tendencia que se invierte en cursos más avanzados (Alborno \& Gaad, 2014). En el presente estudio, el hecho de que se trate de alumnos de primaria, y de que estos experimenten menos aceptación por parte de sus iguales y menos apoyo por parte de sus profesores, puede estar revelando la falta de preparación de los profesores para utilizar diferentes estrategias de enseñanza que den respuesta a estudiantes con diversidad de condiciones personales y sociales (Amr, Al-Natour, Al-Abdallat y Alkhamra, 2016; Odongo y Davidson, 2016), no necesariamente etiquetados como ACNEAE.

Si bien en este estudio esperábamos que los hallazgos se mantuvieran tanto para alumnos con necesidades específicas de apoyo educativo, como por quienes no las presentaran, los resultados apoyan parcialmente esta predicción. Por un lado hemos encontrado que las valoraciones del centro educativo por parte de los alumnos con NEAE son más similares. Hay que señalar, no obstante, que factores como la práctica ausencia de alumnos Populares en este grupo, así como el menor tamaño de las muestras, pueda estar contribuyendo a explicar estos resultados. Por otro lado, es posible que la presencia de necesidades educativas actúe como factor homogeneizador de las percepciones. Pero además, cuando se comparan las diferencias entre grupos sociométricos y presencia o ausencia de NEAE, se aprecia que los alumnos olvidados con NEAE perciben que reciben significativamente más atención por parte de sus profesores. Estas diferencias podrían estar 
reflejando la atención específica que dichos alumnos reciben del profesor de apoyo. Al respecto, las estrategias de agrupamiento que adoptan las escuelas, en forma de atención especializada fuera del aula, podrían estar afianzando la dualidad educación general versus educación especial, más que potenciando la inclusión. Es decir, el apoyo especializado que se presta hace que la responsabilidad de la enseñanza de los alumnos con NEAE recaiga sobre los profesores de educación especial (Amr et al., 2016; Ávila, 2017), lo que a su vez, hace que el profesor general se sienta responsable de la enseñanza de solo una parte de alumnos. Esto es de interés porque, como sabemos, contar con recursos de apoyo o con recursos potenciales para la inclusión no garantiza la obtención de unos resultados inclusivos (Saloviita, 2020). Por último, cabe destacar que en el presente estudio tan solo un alumno con NEAE obtuvo una valoración sociométrica de Popular, lo cual puede ser considerado como un indicador más del bajo nivel de aceptación de estos estudiantes. En conclusión, el presente estudio permite afirmar que:

1. Los alumnos sin NEAE son objeto de más elecciones por sus iguales en el grupo-clase, y por lo tanto, su estatus sociométrico es predominantemente de Entrañable o Popular; mientras que los alumnos con NEAE son objeto de más rechazo y su estatus sociométrico es predominantemente de Olvidado o Rechazado.

2. Los alumnos se diferencian significativamente en la percepción que tienen de sus centros educativos. Dicha percepción se ve afectada significativamente por las elecciones y rechazos que reciben de sus iguales en el grupo-clase.

3. Los alumnos aceptados por sus iguales (populares o entrañables) muestran mayores niveles de satisfacción con el centro educativo, mientras que quienes no experimentan tal aceptación (olvidados o rechazados) muestran menos satisfacción con su centro educativo.

\section{Implicaciones}

A nivel práctico, los resultados de este trabajo empírico apuntalan la importancia de dar voz a los estudiantes como forma de promover procesos significativos de inclusión. De ahí, la importancia de incluir la percepción de los estudiantes de sus centros educativos como indicador de inclusión educativa, y la necesidad de evaluación periódica de la inclusión como un indicador de calidad educativa. 
Una vez más se pone de manifiesto cómo determinadas decisiones organizativas adoptadas por los centros educativos influyen y afectan la aceptación de los estudiantes con discapacidad por sus iguales de desarrollo típico. En todo caso, es conocido que ejemplos de buenas prácticas y de compromiso educativo con la inclusión por parte del profesorado ofrecen sus frutos incluso en casos de NEE más significativas en las que la implementación de actividades que promueven el juego, aprendizaje y participación son claves para obtener cambios significativos (Scholes, Lunn Brownlee, Walker, Johansson, Lawson y Mascadri, 2017) para todos los alumnos. Estos resultados destacan además la importancia de formar a los futuros maestros en la puesta en práctica de estrategias inclusivas en las aulas para así ayudar a reducir la brecha entre la teoría y la práctica (Moliner et al., 2020). La puesta en marcha de estrategias formativas basadas en el diseño integrado para la adquisición de competencias en materia de pedagogía inclusiva ha demostrado ofrecer buenos resultados (Lancaster \& Bain, 2019).

\section{Limitaciones}

Este estudio analiza las percepciones de estudiantes de Educación Primaria. Pese a lo amplio de la muestra, el procedimiento de selección y procedencia hace que los resultados no puedan generalizarse a estudiantes españoles de otros niveles educativos (Educación Secundaria, Bachillerato y Educación Superior). El relativamente limitado número de alumnos con NEAE ha impedido realizar un análisis más pormenorizado de posibles diferencias en función de condiciones personales o sociales de los alumnos. Futuras investigaciones deberían confirmar si los resultados obtenidos difieren o se mantienen en otros niveles educativos, para las diferentes condiciones de los alumnos y ante distintos estatus sociométricos. Por otro lado, el estudio se basa en una metodología cuantitativa y en el empleo de cuestionarios. Estrategias adicionales de recogida de información como la observación directa, pueden permitir recoger información más detallada sobre las conductas e interacciones entre todos los implicados. Por último, posteriores trabajos podrían indagar en las condiciones personales de los alumnos asociadas a una mayor o menor aceptación, como por ejemplo sus habilidades sociales, con objeto de poner en marcha medidas educativas que garanticen la plena igualdad de oportunidades y participación.

\section{AGRADECIMIENTOS}

Nuestro agradecimiento a los 43 tutores de los grupos-clase participantes que hicieron posible el desarrollo de este estudio. 


\section{REFERENCIAS BIBLIOGRÁFICAS}

Adams, D., Harris, A., \& Jones, M.S. (2016). Teacher-parent collaboration for an inclusive classroom: Success for every child. Malaysian Online Journal of Educational Sciences, 4(3), 5874. https://mojes.um.edu.my/article/ view/12657/8148

Ainscow, M. (2016). Diversity and equity: A global education challenge. New Zealand. Journal of Educational Studies, 51(2), 143-155. https://doi. org/10.1007/s40841-016-0056-x

Akin, D., \& Huang, L. M. (2019). Perceptions of college students with disabilities. Journal of Postsecondary Education and Disability, 32(1), 21-33. http://www.ahead.org/publications/ jped

Alborno, N. E., \& Gaad, E. (2014). "Index for inclusion": A framework for school review in the United Arab Emirates. British Journal of Special Education, 41(3), 231-248. http:// dx.doi.org/10.1111/1467-8578.12073

Amr, M., Al-Natour, M., Al-Abdallat, B., \& Alkhamra, H. (2016). Primary school teachers's knowledge, attitudes and views on barriers to inclusion in Jordan. International Journal of Special Education, 31(1), 67-77. https://files. eric.ed.gov/fulltext/EJ1099989.pdf

Ávila, M. (2017). Calidad y equidad educativa: La educación inclusiva como derecho. https://bit.ly/3mpR5Ij

Baydik, B., \& Bakkaloglu, H. (2009). Predictors of sociometric status for low socioeconomic status elementary mainstreamed students with and without special needs. Educational Sciences: Theory and Practice, 9(2), 435-47. https://files.eric.ed.gov/fulltext/ EJ847760.pdf
Blandon, A. Y., Calkins, S. D., Grimm, K. J., Keane, S. P., \& O’Brien, M. (2010). Testing a developmental cascade model of emotional and social competence and early peer acceptance. Development and Psychopathology, 22(1), 737-748. https://doi.org/10.1017/ S0954579410000428

Booth, T., Ainscow, M., \& Kingston, D. (2006). Index for Inclusion: developing play, learning, and participation in early years and childcare. Centre for Studies on Inclusive Education (CSIE). https:// bit.ly/3DjEr4J

Crouch, R., Keys, C. B., \& McMahon, S. D. (2014). Student-teacher relationships matter for school inclusion: School belonging, disability, and school transitions. Journal of Prevention and Intervention in the Community, 42 (1), 20-30. https://doi.org/10.1080/1085235 2.2014 .855054

DeRosier, M., \& Thomas, J. M. (2003). Strengthening sociometric prediction: scientific advances in the assessment of children's peer relations. Child Development, 74(5), 1379-1392. https:// doi.org/10.1111/1467-8624.00613

De Vroey, A., Struyf, E., \& Petry, K. (2016). Secondary schools included: A literature review. International Journal of Inclusive Education, 20(2), 109-135. https://doi.org/10.1080/13603116.2015 .1075609

Díaz-Aguado, M.J. (2006). Del acoso escolar a la cooperación en las aulas. Pearson.

García-Bacete, F. J. (2006). La identificación de los alumnos rechazados. Comparación de métodos sociométricos de nominaciones bidimensionales. Infancia y 
Aprendizaje, 29(4), 437-451. https://doi. org/10.1174/021037006778849585

González-Gil, F., Gómez-Vela, M., \& Jenaro, C. (2007). Traducción y adaptación del Index para la inclusión. Desarrollo del juego, el aprendizaje y la participación en educación infantil. Centre for Studies on Inclusive Education (CSIE). https://bit. ly/3AeNDFx

Haug, P. (2017). Understanding inclusive education: ideals and reality. Scandinavian Journal of Disability Research, 19(3), 206-217. https://doi.or $\mathrm{g} / 10.1080 / 15017419.2016 .1224778$

Héctor, S., \& Olivarez, A. (1995). A metaanalysis of peer rating sociometric studies with pupils with learning disabilities. The Journal of Special Education, 29(1), 1-19. http://dx.doi. org/10.1177/002246699502900101

Hodson, P., Baddeley, A., Laycock, S., \& Williams, S. (2005). Helping Secondary Schools to Be More Inclusive of Year 7 Pupils with SEN. Educational Psychology in Practice, 21(1), 53-67.

Krampač, A., \& Kolak, A. (2018). Peer relations in inclusive classes. Research in Pedagogy, 8(1), 17-35. http://dx.doi. org/10.17810/2015.68

Kwon, K., Hong, S., \& Jeon, H. (2017). Classroom readiness for successful inclusion: Teacher factors and preschool children's experience with and attitudes toward peers with disabilities. Journal of Research in Childhood Education, 31(3), 360-378. http://dx.doi.org/10.1080/02568543.20 17.1309480

Lancaster, J., \& Bain, A. (2019). Designing university courses to improve preservice teachers' pedagogical content knowledge of evidence-based inclusive practice. Australian Journal of Teacher Education, 44(2), 51-65.

Ley Orgánica 2/2006, de 3 de mayo, de Educación (LOE). Boletín Oficial del Estado, 4 de mayo de 2006, núm. 106, 1-112. https://www.boe.es/ buscar/pdf/2006/BOE-A-2006-7899consolidado.pdf

Lindner, K., Alnahdi, G., Wahl, S., \& Schwab, S. (2019). Personalization teaching approaches in inclusive classrooms: perspectives of students and teachers. Frontiers in Education, 4(58), 1-13. http://dx.doi.org/10.3389/ feduc. 2019.00058

Martín, E., Muñoz de Bustillo, M.C., \& Pérez, N. (2011). Las relaciones de amistad en la escuela de los menores en acogimiento residencial. Revista de Psicodidáctica, 6(2), 351-366. https://www.redalyc.org/ pdf/175/17518828005.pdf

Moliner, O., Arnaiz, P., \& Sanahuja, A. (2020). Rompiendo la brecha entre teoría y práctica: ¿Qué estrategias utiliza el profesorado universitario para movilizar el conocimiento sobre educación inclusiva? Educación XX1, 23(1), 173-195. https://doi.org/10.5944/ educXX1.23753

Nepi, L. D., Fioravanti, J., Nannini, P., \& Peru, A. (2015). Social acceptance and the choosing of favourite classmates: a comparison between students with special educational needs and typically developing students in a context of full inclusion. British Journal of Special Education, 42(3), 320-337. http:// dx.doi.org/10.1111/1467-8578.12096

$\mathrm{Ng}$, C. K. M., \& Yuen, M. (2011). The perceived school climate in invitational schools in Hong Kong: Using the Chinese version of the Inviting School Survey-Revised (ISSR). Journal of Invitational Theory and 
Practice, 17, 11-21. https://files.eric. ed.gov/fulltext/EJ974509.pdf

Odongo, G., \& Davidson, R. (2016). Examining the attitudes and concerns of the Kenyan teachers toward the inclusion of children with disabilities in the general education classroom: A mixed methods study. International Journal of Special Education, 31(2), 1-30. https://files.eric.ed.gov/fulltext/ EJ1111087.pdf

Perolli-Shehu, B. (2019). Peer acceptance in early childhood: Links to socio-economic status and social competence, Journal of Social Studies Education Research, 10(4), 176200. https://files.eric.ed.gov/fulltext/ EJ1241768.pdf

Pijl, S. J., Frostad, P., \& Flem, A. (2008). The social position of pupils with special needs in regular schools. Scandinavian Journal of Educational Research, 52(4), 387-405. https://doi. org/10.1080/00313830802184558

Prince, E. J., \& Hadwin, J. (2013). The role of a sense of school belonging in understanding the effectiveness of inclusion of children with special educational needs. International Journal of Inclusive Education, 17(3), 238-262. http://dx.doi.org/10.1080/136 03116.2012 .676081

Purkey, W., \& Novak, J. (2016). Fundamentals of invitational education ( $2^{\text {nd }}$ ed.). International Alliance for Invitational Education.

Ramberg, J., \& Watjins, A. (2020). Exploring inclusive education across Europe: some insights from the European agency statistics on inclusive education. FIRE, Forum for International Research in Education, 6(1), 85-101. https://doi.org/10.32865/ fire202061172
Rose, K. K., Barahona, D., \& Muro, J. (2017). Peer perceptions of students receiving pull-out services in elementary school: A multi-age study. International Journal of Inclusive Education, 21(4), 376-388. http:// dx.doi.org/10.1080/13603116.2016.11 97323

Rose, R., \& Shevlin, M. (2017). A sense of belonging: Children's views of acceptance in "inclusive" mainstream schools, International Journal of Whole Schooling, Special Issue, 13(1), 65-80. https://eric.ed.gov/?id=EJ1142322

Saloviita, T. (2020) Attitudes of teachers towards inclusive education in Finland, Scandinavian Journal of Educational Research, 64(2) 270-282. http://dx.doi.org/10.1080/00313831.20 18.1541819

Scholes, L., Lunn Brownlee, J., Walker, S., Johansson, E., Lawson, V., \& Mascadri, J. (2017). Promoting social inclusion in the early years of elementary school: a focus on children's epistemic beliefs for moral reasoning. International Journal of Inclusive Education, 21(5), 507-520. https://doi.org/10.1080/13603116.2016 .1223181

Schwab, S. (2018). Friendship stability among students with and without special educational needs. Educational Studie, 45(3), 390-401. http://dx.doi.or $\mathrm{g} / 10.1080 / 03055698.2018 .1509774$.

Schwab, S., Sharma, U., \& Hoffmann, L. (2019). How inclusive are the teaching practices of my German, Maths and English teachers? Psychometric properties of a newly developed scale to assess personalisation and differentiation in teaching practices. International Journal of Inclusive Education, 1-20. http://dx.doi.org/10.1 080/13603116.2019.1629121 
Shogren, K. A., Gross, J.M., ForberPratt, A.J., Francis, G. L. Satter, A., Blue-Banning, M., \& Hill, C. (2015). The perspectives of students with and without disabilities on inclusive schools. Research and Practice for Persons with Severe Disabilities, 40(4), 243-260. http://dx.doi. org/10.1177/1540796915583493

Stites, M., Rakes, C., Noggle, A., \& Sha, S. (2018). Preservice teacher perceptions of preparedness to teach in inclusive settings as an indicator of teacher preparation program effectiveness. Discourse and Communication for Sustainable Education, 9(2), 21-39. http://dx.doi.org/10.2478/dcse-20180012

Van der Wilt, F., Van der Veen, Ch., Van Kruistum, C., \& Van Oers, B. (2020). Language abilities and peer rejection in kindergarten: A mediation analysis. Early Education and Development, 31(2), 269-283. https://doi.org/ 10.1080/10409289.2019.1624145
Vignes, C., Godeau, E., Sentenac, M., Coley, N., Navarro, F., Grandjean, M., \& Arnaud, C. (2009). Determinants of students' attitudes towards peers with disabilities. Developmental Medicine and Child Neurology, 51, 473479. https://doi.org/10.1111/j.14698749.2009.03283.x

Wallace, T. L., Kelcey, B., \& Ruzek, E. (2016). What can student perception surveys tell us about teaching? Empirically testing the underlying structure of the tripod student perception survey. American Educational Research Journal, 53, 1834-1868. https://doi. org/10.3102/0002831216671864

Wendelborg, C., \& Tossebro, J. (2011). Educational arrangements and social participation with peers amongst children with disabilities in regular schools. International Journal of Inclusive Education, 15(5), 497-512. https://doi. org/10.1080/13603110903131739 


\section{PERFIL ACADÉMICO Y PROFESIONAL DE LOS AUTORES}

Margarita Rodríguez Gudiño. ORCID: https://orcid.org/0000-0002-3941$242 \mathrm{X}$

Licenciada en psicología por la UNED, Máster en Integración de Personas con Discapacidad: Habilitación y Rehabilitación. INICO, Universidad de Salamanca y Máster en Trastornos de la Comunicación: Neurociencia de la Audición y Lenguaje, Universidad de Salamanca. Es profesora de enseñanza secundaria, especialidad psicología y pedagogía y orientadora en el Equipo de Orientación Educativa y Psicopedagógica (EOEP) de Olivenza (Badajoz). E-mail: margarigudi@hotmail.com

Cristina Jenaro Río. ORCID: https://orcid.org/0000-0003-2060-1658

Catedrática de universidad desde 2016, del departamento de personalidad, evaluación y tratamiento psicológicos de la facultad de psicología, Universidad de Salamanca. Es investigadora del INICO, Instituto Universitario de Integración en la Comunidad y miembro de la Unidad de Investigación Consolidada (UIC) y reconocida por la Junta de Castilla y León el 22 de septiembre de 2015. Es también miembro del equipo de investigación NEUR-09 Autismo y Trastornos del Neurodesarrollo. Infoautismo, del IBSAL. Sus principales líneas de investigación se relacionan con la discapacidad, inclusión, salud, calidad de vida, actitudes y transición a la vida adulta. E-mail: crisje@usal.es

Raimundo Castaño Calle. ORCID: https://orcid.org/0000-0003-1586-6491

Profesor titular desde 2018, de la Facultad de Educación de la Universidad Pontificia de Salamanca. Máster en Actividad Físico-Deportiva e Integración Social. Actual vicedecano de profesorado y títulos de dicha facultad. Miembro del equipo de investigación EGIIOFYD, Enseñanza, Gestión, Intervención, Inclusión y Orientación de la Actividad Física y del Deporte. Miembro del Comité de Revisores Científicos de la Revista Comunicar (Indexed in JCR (Q1); SCOPUS (Q1); Google Scholar (1st top 100). Entre sus líneas de investigación se encuentran la atención a la diversidad y la educación inclusiva, el alumnado con Necesidades Específicas de Apoyo Educativo o la Inclusión en el marco de la actividad física y el deporte. E-mail: rcastanoca@upsa.es

Fecha Recepción del Artículo: 04. Marzo. 2021

Fecha Modificación del Artículo: 01. Mayo. 2021

Fecha Aceptación del Artículo: 19. Mayo. 2021

Fecha Revisión para Publicación: 09. Septiembre. 2021 